\title{
Semisimplification for subgroups of reductive algebraic groups
}

\author{
Michael Bate ${ }^{1}$, Benjamin Martin ${ }^{2}$ and Gerhard Röhrle ${ }^{3}$ \\ ${ }^{1}$ Department of Mathematics, University of York, York YO10 5DD, United Kingdom; E-mail: michael.bate@york.ac.uk. \\ ${ }^{2}$ Department of Mathematics, University of Aberdeen, King's College, Fraser Noble Building, Aberdeen AB24 3UE, \\ United Kingdom; E-mail: b.martin@abdn.ac.uk. \\ ${ }^{3}$ Fakultät für Mathematik, Ruhr-Universität Bochum, Universitätsstraße 150, D-44780 Bochum, Germany; \\ E-mail: gerhard.roehrle@rub.de.
}

Received: 28 May 2020; Revised: 3 July 2020; Accepted: 21 July 2020

2020 Mathematics Subject Classification: 20G15 (primary); 14L24 (secondary)

Keywords: semisimplification; $G$-complete reducibility; geometric invariant theory; rationality; cocharacter-closed orbits; degeneration of $G$-orbits

\begin{abstract}
Let $G$ be a reductive algebraic group—possibly non-connected—over a field $k$, and let $H$ be a subgroup of $G$. If $G=\mathrm{GL}_{n}$, then there is a degeneration process for obtaining from $H$ a completely reducible subgroup $H^{\prime}$ of $G$; one takes a limit of $H$ along a cocharacter of $G$ in an appropriate sense. We generalise this idea to arbitrary reductive $G$ using the notion of $G$-complete reducibility and results from geometric invariant theory over non-algebraically closed fields due to the authors and Herpel. Our construction produces a $G$-completely reducible subgroup $H^{\prime}$ of $G$, unique up to $G(k)$-conjugacy, which we call a $k$-semisimplification of $H$. This gives a single unifying construction that extends various special cases in the literature (in particular, it agrees with the usual notion for $G=\mathrm{GL}_{n}$ and with Serre's ' $G$-analogue' of semisimplification for subgroups of $G(k)$ from [19]). We also show that under some extra hypotheses, one can pick $H^{\prime}$ in a more canonical way using the Tits Centre Conjecture for spherical buildings and/or the theory of optimal destabilising cocharacters introduced by Hesselink, Kempf, and Rousseau.
\end{abstract}

\section{Contents}

1 Introduction 1

2 Cocharacter-closed orbits $\quad 2$

$3 G$-complete reducibility $\quad 4$

4 k-semisimplification $\quad 5$

5 Optimality and normal subgroups $\quad 7$

\section{Introduction}

The aim of this paper is to present a construction of the semisimplification of a subgroup $H$ of a (possibly non-connected) reductive linear algebraic group $G$ over an arbitrary field $k$. This construction unifies and generalizes many concepts already in the literature within a single framework. For example, the semisimplification of a module for a group is a well-known construction in representation theory, corresponding in our case to the situation where $H \subseteq \mathrm{GL}_{n}(k)$. Building on this idea, for $G$, a connected 
reductive linear algebraic group over a field $k$, and $H$, a subgroup of $G(k)$, Serre introduced the concept of a ' $G$-analogue' of semisimplification from representation theory in [19, Section 3.2.4]. This notion is also used for representations of various kinds of algebras: for example, see [12], [8], [16], [23], and [24]. It is also an ingredient in work of Lawrence-Sawin on the Shafarevich Conjecture for abelian varieties [13] and work of Lawrence-Venkatesh on Mordell's Conjecture [14], which involve Galois representations taking values in possibly non-connected reductive $p$-adic groups.

We begin by recalling how the most basic case works. Let $n \in \mathbb{N}$, and let $H$ be a subgroup of $\mathrm{GL}_{n}(k)$. There is an $H$-module filtration of $k^{n}$ such that the successive quotients are irreducible, by the Jordan-Hölder Theorem. In terms of matrices, this implies that by changing basis if necessary, we may assume that $H$ is in upper block-triangular form, with the action of $H$ on each quotient being represented by the corresponding block on the diagonal. Letting $H^{\prime}$ be the subgroup of $\mathrm{GL}_{n}(k)$ consisting of the block diagonal matrices obtained by taking each element of $H$ and replacing the entries above the block diagonal with $0 \mathrm{~s}$, we obtain a subgroup that acts semisimply on $k^{n}$ - that is, $H^{\prime}$ is completely reducible. Since this construction is independent of the choice of basis up to $\mathrm{GL}_{n}(k)$-conjugacy, again by the Jordan-Hölder Theorem, it is reasonable to call $H^{\prime}$ the semisimplification of $H$.

We now explain several of the ingredients of our construction in the case that $k$ is algebraically closed, which removes some technicalities. Recall $[2,19]$ that if $G$ is connected and $H$ is a subgroup of $G$, then $H$ is $G$-completely reducible ( $G$-cr for short) if for any parabolic subgroup $P$ of $G$ such that $P$ contains $H$, there is a Levi subgroup $L$ of $P$ such that $L$ contains $H$. If $G=\mathrm{GL}_{n}$, then $H$ is $G$-cr if and only if $k^{n}$ is completely reducible as an $H$-module; this follows from the usual characterisation of parabolic subgroups of $\mathrm{GL}_{n}$ as stabilizers of flags of subspaces. We make the same definition for arbitrary reductive $G$, replacing parabolic subgroups and Levi subgroups with R-parabolic subgroups and R-Levi subgroups instead (see Section 2 for details).

To perform our construction, we apply a characterisation of $G$-complete reducibility in terms of geometric invariant theory (GIT). We see this idea already in our original example: we can view $H^{\prime}$ as a degeneration of $H$ in the following sense. Let the sizes of the blocks down the diagonal be $n_{1}, \ldots, n_{r}$, and define a cocharacter $\lambda: \mathbb{G}_{m} \rightarrow \mathrm{GL}_{n}$ by

$$
\lambda(a)=\operatorname{diag}\left(a^{r}, \ldots, a^{r}, \ldots, a^{1}, \ldots, a^{1}\right), \text { with } n_{i} \text { occurrences of } a^{r-i+1}, 1 \leq i \leq r .
$$

For each $a \in k^{*}$, define $H_{a}=\lambda(a) H \lambda(a)^{-1}$ for $a \in k^{*}$. Then $H^{\prime}=\lim _{a \rightarrow 0} H_{a}$ in an appropriate sense.

Our definition of $k$-semisimplification (Definition 4.1) for arbitrary $k$ is new, generalizes the one given by Serre in [19, Section 3.2.4], and is closely related to the definition given in [6] using optimal destabilising cocharacters; the two notions agree whenever the latter makes sense (see also [15, Section 4] for the algebraically closed case). We prove that the $k$-semisimplification of a subgroup $H$ of $G$ is unique up to conjugacy (Theorem 4.5), generalizing [19, Proposition 3.3(b)]. In Theorem 5.4, we show that a normal subgroup of a $G$-completely reducible subgroup $H$ is $G$-completely reducible and that the process of $k$-semisimplification behaves well under passing to normal subgroups of $H$, if $k$ is perfect or $G$ is connected. The proof rests on deep results from the theory of spherical buildings and the Hesselink-Kempf-Rousseau theory of optimal destabilising cocharacters. We give a short and selfcontained exposition, bringing together some results (such as Corollary 3.5) that follow from previous work but are not easily extracted from earlier papers.

\section{Cocharacter-closed orbits}

Following [7] and our earlier work [6, 1], we regard an affine variety over a field $k$ as a variety $X$ over the algebraic closure $\bar{k}$ together with a choice of $k$-structure. We denote the separable closure of $k$ by $k_{s}$. We write $X(k)$ for the set of $k$-points of $X$ and $X(\bar{k})$ (or just $X$ ) for the set of $\bar{k}$-points of $X$. By a subvariety of $X$, we mean a closed $k$-subvariety of $X$; a $k$-subvariety is a subvariety that is defined over $k$. We denote by $M_{n}$ the associative algebra of $n \times n$ matrices over $k$. Below $G$ denotes a possibly nonconnected reductive linear algebraic group over $k$. By a subgroup of $G$, we mean a closed $\bar{k}$-subgroup; 
and by a $k$-subgroup, we mean a subgroup that is defined over $k$. (We note here that much of what follows works for non-closed subgroups-most of the important conditions hold for $H$ if and only if they hold for the Zariski closure $\bar{H}$; the details are left to the reader.) By $G^{0}$, we denote the identity component of $G$, and likewise for subgroups of $G$.

We define $Y_{k}(G)$ to be the set of $k$-defined cocharacters of $G$ and $Y(G):=Y_{\bar{k}}(G)$ to be the set of all cocharacters of $G$.

Let $H$ be a subgroup of $G$. Even if $H$ is $k$-defined, the (set-theoretic) centralizer $C_{G}(H)$ need not be $k$-defined in general. It is useful to have criteria to ensure that $C_{G}(H)$ is $k$-defined (see Proposition 3.4 and Section 5). For instance, if $k$ is perfect and $H$ is $k$-defined, then $C_{G}(H)$ is $k$-defined. We say that $H$ is separable if the scheme-theoretic centralizer $\mathscr{C}_{G}(H)$ is smooth [2, Definition 3.27]; for instance, any subgroup of $\mathrm{GL}_{n}$ is separable [2, Example 3.28] (see [5] for more examples of separable subgroups). If $H$ is $k$-defined and separable, then $C_{G}(H)$ is $k$-defined (see [1, Proposition 7.4]).

Next we recall some basic notation and facts concerning parabolic subgroups in (non-connected) reductive groups $G$ from [2, Section 6] and [6]. Given $\lambda \in Y(G)$, we define

$$
P_{\lambda}=\left\{g \in G \mid \lim _{a \rightarrow 0} \lambda(a) g \lambda(a)^{-1} \text { exists }\right\}
$$

and $L_{\lambda}=C_{G}(\operatorname{Im}(\lambda))$ (for the definition of a limit, see [20, Section 3.2.13]). We call $P_{\lambda}$ an $R$-parabolic subgroup of $G$ and $L_{\lambda}$ an $R$-Levi subgroup of $P_{\lambda}$; they are subgroups of $G$. We have $P_{\lambda}=L_{\lambda}=G$ if $\operatorname{Im}(\lambda)$ is contained in the centre of $G$. For ease of reference, we record without proof some basic facts about these subgroups.

\section{Lemma 2.1.}

(i) If $P$ is a $k$-defined $R$-parabolic subgroup, then $R_{u}(P)$ is $k$-defined.

(ii) If $P$ is a parabolic subgroup of $G^{0}$, then the normalizer $N_{G}(P)$ is an $R$-parabolic subgroup of $G$, and $N_{G}(P)$ is $k$-defined if $P$ is.

If $G$ is connected, then every pair $(P, L)$ consisting of a parabolic $k$-subgroup $P$ of $G$ and a Levi $k$-subgroup $L$ of $P$ is of the form $(P, L)=\left(P_{\lambda}, L_{\lambda}\right)$ for some $\lambda \in Y_{k}(G)$, and vice versa [20, Lemma 15.1.2(ii)]. In general, if $\lambda \in Y_{k}(G)$, then $P_{\lambda}$ and $L_{\lambda}$ are $k$-defined [6, Lemma 2.5], but the converse is not so straightforward. If $P$ is an R-parabolic $k$-subgroup and $L$ is an R-Levi $k$-subgroup of $P$, then for any maximal $k$-torus $T$ of $L$, there exists $\lambda \in Y_{k_{s}}(T)$ such that $P=P_{\lambda}$ and $L=L_{\lambda}$. However, it is possible that $P$ is a $k$-defined R-parabolic subgroup and yet there does not exist any $\mu \in Y_{k}(G)$ such that $P=P_{\mu}$, and similarly for R-Levi subgroups—see [6, Remark 2.4]. This complicates some of the arguments below.

Lemma 2.2. Let $P$ be an R-parabolic subgroup of $G$ and $L$ an $R$-Levi subgroup of $P$.

(i) We have $P \cong L \ltimes R_{u}(P)$, and this is a $k$-isomorphism if $P$ and $L$ are $k$-defined.

(ii) Any two R-Levi $k$-subgroups of an R-parabolic $k$-subgroup $P$ are $R_{u}(P)(k)$-conjugate.

We denote the canonical projection from $P$ to $L$ by $c_{L}$; this is $k$-defined if $P$ and $L$ are. If we are given $\lambda \in Y(G)$ such that $P=P_{\lambda}$ and $L=L_{\lambda}$, then we often write $c_{\lambda}$ instead of $c_{L}$. We have $c_{\lambda}(g)=\lim _{a \rightarrow 0} \lambda(a) g \lambda(a)^{-1}$ for $g \in P_{\lambda}$; the kernel of $c_{\lambda}$ is the unipotent radical $R_{u}\left(P_{\lambda}\right)$, and the set of fixed points of $c_{\lambda}$ is $L_{\lambda}$.

Let $m \in \mathbb{N}$. Below we consider the action of $G$ on $G^{m}$ by simultaneous conjugation: $g \cdot\left(g_{1}, \ldots, g_{m}\right)=$ $\left(g g_{1} g^{-1}, \ldots, g g_{m} g^{-1}\right)$. Given $\lambda \in Y(G)$, we have a map $P_{\lambda}^{m} \rightarrow L_{\lambda}^{m}$ given by $\mathbf{g} \mapsto \lim _{a \rightarrow 0} \lambda(a) \cdot \mathbf{g}$; we abuse notation slightly and also call this map $c_{\lambda}$. For any $\mathbf{g} \in P_{\lambda}^{m}$, there exists an R-Levi $k$-subgroup $L$ of $P_{\lambda}$ with $\mathbf{g} \in L^{n}$ if and only if $c_{\lambda}(\mathbf{g})=u \cdot \mathbf{g}$ for some $u \in R_{u}\left(P_{\lambda}\right)(k)$.

Our main tool from GIT is the notion of cocharacter-closure, introduced in [6] and [1].

Definition 2.3. Let $X$ be an affine $G$-variety and let $x \in X$ (we do not require $x$ to be a $k$-point). We say that the orbit $G(k) \cdot x$ is cocharacter-closed over $k$ if for all $\lambda \in Y_{k}(G)$ such that $x^{\prime}:=\lim _{a \rightarrow 0} \lambda(a) \cdot x$ exists, $x^{\prime}$ belongs to $G(k) \cdot x$. If $k=\bar{k}$ then it follows from the Hilbert-Mumford Theorem that $G(k) \cdot x$ is cocharacter-closed over $k$ if and only if $G(k) \cdot x$ is closed [11, Theorem 1.4]. If $\mathcal{O}$ is a $G(k)$-orbit in $X$, 
then we say that $\mathcal{O}$ is accessible from $x$ over $k$ if there exists $\lambda \in Y_{k}(G)$ such that $x^{\prime}:=\lim _{a \rightarrow 0} \lambda(a) \cdot x$ belongs to $\mathcal{O}$.

Example 2.4. If $X=G^{m}, \lambda \in Y_{k}(G)$, and $\mathbf{g} \in P_{\lambda}^{m}$, then $G(k) \cdot c_{\lambda}(\mathbf{g})$ is accessible from $\mathbf{g}$ over $k$.

The following result is [1, Theorem 1.3].

Theorem 2.5 (Rational Hilbert-Mumford Theorem). Let $G, X, x$ be as above. Then there is a unique $G(k)$-orbit $\mathcal{O}$ such that $\mathcal{O}$ is cocharacter-closed over $k$ and accessible from $x$ over $k$.

\section{3. $G$-complete reducibility}

Definition 3.1. Let $H$ be a subgroup of $G$. We say that $H$ is $G$-completely reducible over $k$ ( $G$-cr over $k$ ) if for any $R$-parabolic $k$-subgroup $P$ of $G$ such that $P$ contains $H$, there is an $R$-Levi $k$-subgroup $L$ of $P$ such that $L$ contains $H$. We say that $H$ is $G$-irreducible over $k(G$-ir over $k)$ if $H$ is not contained in any proper $R$-parabolic $k$-subgroup of $G$ at all.

Remark 3.2. We say that $H$ is $G$-cr if $H$ is $G$-cr over $\bar{k}-c f$. Section 1. More generally, if $k^{\prime} / k$ is an algebraic field extension, then we may regard $G$ as a $k^{\prime}$-group, and it makes sense to ask whether $H$ is $G$-cr over k'.

For more on $G$-complete reducibility, see [18, 19, 2].

Note that the definitions make sense even if $H$ is not $k$-defined. It is immediate that $G$-irreducibility over $k$ implies $G$-complete reducibility over $k$. We have $P_{g \cdot \lambda}=g P_{\lambda} g^{-1}$ and $L_{g \cdot \lambda}=g L_{\lambda} g^{-1}$ for any $\lambda \in Y(G)$ and any $g \in G$ (see, for example, [2, Section 6]). It follows that if $H$ is $G$-cr over $k$ (respectively, $G$-ir over $k$ ), then so is any $G(k)$-conjugate of $H$. More generally, one can show that if $H$ is $G$-cr over $k$ (respectively, $G$-ir over $k$ ), then so is $\phi(H)$ for any $k$-defined automorphism $\phi$ of $G$. If $k=\bar{k}$ and $H$ is $G$-cr, then $H$ is reductive [19, Proposition 4.1] and [2, Section 2.4, Section 6.2]. It follows from Proposition 3.4 below that if $H$ is $k$-defined, $k$ is perfect and $H$ is $G$-cr over $k$, then $H$ is reductive. We see below (Corollary 3.5) that the converse holds in characteristic 0. On the other hand, the converse is false in general, as is shown by the example in [22, Proof of Proposition 1.10].

We now explain the link between $G$-complete reducibility and GIT. Fix a $k$-embedding $\iota: G \rightarrow \mathrm{GL}_{n}$ for some $n \in \mathbb{N}$. Let $H$ be a subgroup of $G$. Let $m \in \mathbb{N}$, and let $\mathbf{h}=\left(h_{1}, \ldots, h_{m}\right) \in H^{m}$. We call $\mathbf{h}$ a generic tuple for $H$ with respect to $\iota$ if $h_{1}, \ldots, h_{m}$ generate the subalgebra of $M_{n}$ generated by $H$ [6, Definition 5.4]. Note that we don't insist that $\mathbf{h}$ is a $k$-point. Our constructions below do not depend on the choice of $\iota$, so we suppress the words 'with respect to $\iota$ '. It is immediate that if $\mathbf{h} \in H^{m}$ is a generic tuple for $H$ and $g \in G$, then $g \cdot \mathbf{h}$ is a generic tuple for $g H^{-1}$.

Theorem 3.3 ([1, Theorem 9.3]). Let $H$ be a subgroup of $G$, and let $\mathbf{h} \in H^{m}$ be a generic tuple for $H$. Then $H$ is $G$-completely reducible over $k$ if and only if $G(k) \cdot \mathbf{h}$ is cocharacter-closed over $k$.

Using this result, one can derive many results on $G$-complete reducibility: for instance, see [2] for the algebraically closed case and $[6,1]$ for arbitrary $k$. Note that if $\mathbf{h} \in H^{m}$ is a generic tuple for $H$, then the centralizer $C_{G}(H)$ coincides with the stabilizer $G_{\mathbf{h}}$.

Proposition 3.4. Let $H$ be a $k$-subgroup of $G$. Suppose $k$ is perfect. Then $H$ is $G$-completely reducible over $k$ if and only if $H$ is $G$-completely reducible.

Proof. If $k$ is perfect, then $\bar{k} / k$ is separable and $C_{G}(H)$ is $k$-defined. The result now follows from [1, Corollary 9.7(i)].

Corollary 3.5. Suppose $\operatorname{char}(k)=0$. Let $H$ be a $k$-subgroup of $G$. Then $H$ is $G$-completely reducible over $k$ if and only if $H$ is reductive.

Proof. If $k=\bar{k}$, then this is well known (see [19, Proposition 4.2] and [2, Section 2.2, Section 6.3], for example). The result for arbitrary $k$ now follows from Proposition 3.4. 
Recall that if $S$ is a $k$-split torus of $G$, then $C_{G}(S)$ is an R-Levi $k$-subgroup of $G$ [1, Lemma 2.5]. Part (i) of the next result gives the converse, and part (ii) strengthens [1, Corollary 9.7(ii)]: we do not need the hypotheses that $H$ and $C_{G}(H)$ are $k$-defined. See also [19, Proposition 3.2].

Proposition 3.6. Let $L$ be an $R$-Levi k-subgroup of $G$, and let $H$ be a subgroup of $L$.

(a) There exists a $k$-split torus $S$ in $G$ such that $L=C_{G}(S)$.

(b) $H$ is $G$-completely reducible over $k$ if and only if $H$ is L-completely reducible over $k$.

Proof. (a). We can choose $\lambda \in Y_{k_{s}}(G)$ such that $L=C_{G}(\operatorname{Im}(\lambda))$. Let $\lambda=\lambda_{1}, \lambda_{2}, \ldots, \lambda_{r} \in Y_{k_{s}}(G)$ be the $\operatorname{Gal}\left(k_{s} / k\right)$-conjugates of $\lambda$, and let $S$ be the subtorus of $Z(L)^{0}$ generated by the subtori $\operatorname{Im}\left(\lambda_{i}\right)$. Then $S$ is $k$-defined, and $L=C_{G}(S)$. The product map $\lambda_{1} \times \cdots \times \lambda_{r}$ gives an epimorphism from $\bar{k}^{*} \times \cdots \times \bar{k}^{*}$ onto $S$. But a quotient of a split $k$-torus is $k$-split [7, Corollary III.8.4], so $S$ is split.

(b). Given (a), the result now follows from Theorem 3.3 together with [1, Theorem 5.4(ii)].

We finish the section with some results involving non-connected reductive groups that are needed in the sequel. Note that if $Q$ is an R-parabolic $k$-subgroup of $G$ and $M$ is an R-Levi $k$-subgroup of $Q$, then $Q^{0}$ is a parabolic $k$-subgroup of $G^{0}$, and $M^{0}$ is a Levi $k$-subgroup of $Q^{0}$; see [2, Section 6].

Lemma 3.7. Let $P$ be an $R$-parabolic subgroup of $G$, and let $T$ be a maximal torus of $P$. Then there is a unique $R$-Levi subgroup $L$ of $P$ such that $T \subseteq L$. If $P$ and $T$ are $k$-defined, then $L$ is $k$-defined.

Proof. The first assertion is [2, Corollary 6.5]. For the second, suppose $P$ and $T$ are $k$-defined. Then the unique R-Levi subgroup $L$ of $P$ containing $T$ must be Galois-stable and hence $k$-defined also.

\section{Lemma 3.8.}

(a) Let $Q$ be an R-parabolic $k$-subgroup of $G$, and set $P=Q^{0}$. Then the R-Levi $k$-subgroups of $Q$ are precisely the subgroups of the form $N_{Q}(L)$ for $L$, a Levi $k$-subgroup of $P$.

(b) Let $Q, P$ be as in (a), and let $H$ be a subgroup of $P$. Then $H$ is contained in an $R$-Levi $k$-subgroup of $Q$ if and only if $H$ is contained in a Levi $k$-subgroup of $P$. Moreover, if $L$ is a Levi $k$-subgroup of $P$, then $c_{N_{Q}(L)}(H)$ is $N_{Q}(L)$-completely reducible over $k$ if and only if $c_{L}(H)$ is L-completely reducible over $k$.

(c) Let $H$ be a subgroup of $G^{0}$. Then $H$ is $G$-completely reducible over $k$ if and only if $H$ is $G^{0}$ completely reducible over $k$.

Proof. (a) As observed above, if $M$ is an R-Levi subgroup of $Q$, then $M^{0}$ is a Levi subgroup of $P$, and $N_{Q}\left(M^{0}\right)^{0}=N_{P}\left(M^{0}\right)^{0}=M^{0}$. Let $L$ be a Levi subgroup of $P$, and let $T$ be a maximal torus of $L$. By Lemma 3.7 there is a unique R-Levi subgroup $M$ of $Q$ such that $T \subseteq M$. The Levi subgroups $M^{0}$ and $L$ of $P$ both contain $T$, so by Lemma 3.7, they are equal; in particular, $M$ normalizes $L$. Now $N_{Q}(T)$ normalizes $L$ by Lemma 3.7, so $N_{Q}(L)$ meets every component of $Q$. Since $Q=M \ltimes R_{u}(Q), M$ also meets every component of $Q$. It follows that $M=N_{Q}(L)$. Finally, $L$ contains a maximal $k$-torus of $P$ if and only if $N_{Q}(L)$ does, so $L$ is $k$-defined if and only if $N_{Q}(L)$ is, by Lemma 3.7.

(b) The first assertion follows immediately from (a), and part (c) now follows. For the second assertion of (b), note that the restriction of $c_{N_{Q}(L)}(H)$ to $P$ is $c_{L}$; the desired result now follows from part (c) applied to the reductive $k$-group $N_{Q}(L)$.

\section{4. $k$-semisimplification}

Now we come to our main definition.

Definition 4.1. Let $H$ be a subgroup of $G$. We say that a subgroup $H^{\prime}$ of $G$ is a $k$-semisimplification of $H$ (for $G$ ) if there exist an R-parabolic $k$-subgroup $P$ of $G$ and an $R$-Levi $k$-subgroup $L$ of $P$ such that $H \subseteq P$ and $H^{\prime}=c_{L}(H)$, and $H^{\prime}$ is $G$-completely reducible (or equivalently, by Proposition 3.6(ii), $L$-completely reducible) over $k$. We say the pair $(P, L)$ yields $H^{\prime}$. 


\section{Remarks 4.2.}

(a) Let $H$ be a subgroup of $G$. If $H$ is $G$-cr over $k$, then clearly $H$ is a $k$-semisimplification of itself, yielded by the pair $(G, G)$.

(b) Suppose $(P, L)$ yields a $k$-semisimplification $H^{\prime}$ of $H$. Let $L_{1}$ be another $R$-Levi $k$-subgroup of $P$. Then $L_{1}=u L u^{-1}$ for some $u \in R_{u}(P)(k)$, so $c_{L_{1}}(H)=u c_{L}(H) u^{-1}$. Hence $\left(P, L_{1}\right)$ also yields a $k$-semisimplification of $H$. We say that $P$ yields a $k$-semisimplification of $H$.

(c) It is straightforward to check that if $\phi$ is an automorphism of $G$ (as a k-group), $H$ is a subgroup of $G$; and if $(P, L)$ yields a $k$-semisimplification $H^{\prime}$ of $H$, then $\phi\left(H^{\prime}\right)$ is a k-semisimplification of $\phi(H)$, yielded by $(\phi(P), \phi(L))$.

(d) For $G$ connected and $H$ a subgroup of $G(k)$, Definition 4.1 recovers Serre's 'G-analogue' of a semisimplification from [19, Section 3.2.4]. For $k=\bar{k}$, Definition 4.1 generalizes the definition of $\mathcal{D}(H)$ following [15, Lemma 4.1].

Remark 4.3. Let $\mathbf{h}=\left(h_{1}, \ldots, h_{m}\right) \in H^{m}$ be a generic tuple for $H$. Note that $c_{\lambda}$ extends in the obvious way to a homomorphism from a parabolic subalgebra $\mathcal{P}_{\lambda}$ of $M_{n}$ onto a Levi subalgebra $\mathcal{L}_{\lambda}$ of $\mathcal{P}_{\lambda}$, and $\mathcal{P}_{\lambda}$ contains the subalgebra $\mathcal{A}$ generated by $H$. Since the elements $h_{i}$ generate $\mathcal{A}$, the elements $c_{\lambda}\left(h_{i}\right)$ generate $c_{\lambda}(\mathcal{A})$. But $c_{\lambda}(\mathcal{A})$ is the subalgebra of $\mathcal{L}_{\lambda}$ generated by $c_{\lambda}(H)$, so we deduce that $c_{\lambda}(\mathbf{h})=\left(c_{\lambda}\left(h_{1}\right), \ldots, c_{\lambda}\left(h_{m}\right)\right)$ is a generic tuple for $c_{\lambda}(H)$. Hence by Theorem 3.3, $c_{\lambda}(H)$ is a $k$-semisimplification of $H$ if and only if $G(k) \cdot c_{\lambda}(\mathbf{h})$ is cocharacter-closed over $k$. It follows from Theorem 2.5 that $H$ admits at least one $k$-semisimplification: for we can choose $\lambda \in Y_{k}(G)$ such that $G(k) \cdot c_{\lambda}(\mathbf{h})$ is cocharacter-closed over $k$, so $c_{\lambda}(H)$ is a k-semisimplification of $H$, yielded by $\left(P_{\lambda}, L_{\lambda}\right)$.

Lemma 4.4. Suppose that $H^{\prime}$ is a k-semisimplification of $H$. Then there is $\lambda \in Y_{k}(G)$ such that $H^{\prime}$ is yielded by the pair $\left(P_{\lambda}, L_{\lambda}\right)$.

Proof. Suppose $H^{\prime}$ is yielded by the pair $(P, L)$. By the discussion in Section 2, there exist a maximal $k$-torus $T$ of $L$ and $\mu \in Y_{k_{s}}(T)$ such that $P=P_{\mu}$ and $L=L_{\mu}$. Choose a finite Galois extension $k^{\prime} / k$ such that $T$ splits over $k^{\prime}$, and let $\lambda=\sum_{\gamma \in \operatorname{Gal}\left(k^{\prime} / k\right)} \gamma \cdot \mu \in Y_{k}(T)$. One checks easily that $H \subseteq P_{\lambda}$ and $\left.c_{\lambda}\right|_{H}=\left.c_{\mu}\right|_{H}$ (see also the proof of [6, Lemma 2.5(ii)]). Hence $\left(P_{\lambda}, L_{\lambda}\right)$ also yields $H^{\prime}$.

Here is our main result, which was proved in the special case $k=\bar{k}$ in [6, Proposition 5.14(i)]; see also [19, Proposition 3.3(b)]. The uniqueness asserted in Theorem 4.5 is akin to the theorem of Jordan-Hölder.

Theorem 4.5. Let $H$ be a subgroup of $G$. Then any two $k$-semisimplifications of $H$ are $G(k)$-conjugate.

Proof. Let $H_{1}, H_{2}$ be $k$-semisimplifications of $H$. By Lemma 4.4, there exist $\lambda_{1}, \lambda_{2} \in Y_{k}(G)$ such that $\left(P_{\lambda_{1}}, L_{\lambda_{1}}\right)$ realizes $H_{1}$ and $\left(P_{\lambda_{2}}, L_{\lambda_{2}}\right)$ realizes $H_{2}$. Let $\mathbf{h} \in H^{m}$ be a generic tuple for $H$. Then $c_{\lambda_{i}}(\mathbf{h})$ is a generic tuple for $H_{i}$ for $i=1,2$, and each orbit $G(k) \cdot c_{\lambda_{i}}(\mathbf{h})$ is cocharacter-closed over $k$ and accessible from $\mathbf{h}$ over $k$ (Example 2.4). It follows from the uniqueness result in Theorem 2.5 that the closed subset $C_{\mathbf{h}}:=\left\{g \in G \mid g \cdot c_{\lambda_{1}}(\mathbf{h})=c_{\lambda_{2}}(\mathbf{h})\right\}$ contains a $k$-point.

Pick $g \in C_{\mathbf{h}}$. If $H_{2}=g H_{1} g^{-1}$, then we are done. Otherwise, there exists $h \in H$ such that $g c_{\lambda_{1}}(h) g^{-1} \notin H_{2}$ or $g^{-1} c_{\lambda_{2}}(h) g \notin H_{1}$. Without loss, assume the former. We can repeat the above argument, replacing $\mathbf{h}$ with the generic tuple $\mathbf{h}^{\prime}:=(\mathbf{h}, h) \in H^{m+1}$; note that $C_{\mathbf{h}^{\prime}}$ is properly contained in $C_{\mathbf{h}}$. The result now follows by a descending chain condition argument.

Definition 4.6. We define $\mathcal{D}_{k}(H)$ to be the set of $G(k)$-conjugates of any $k$-semisimplification of $H$ (see also the discussion preceding [15, Theorem 1.4]). This is well-defined by Theorem 4.5.

Example 4.7. Let $H$ be a subgroup of $G$. As noted in Remark 4.2(a), if $H$ is $G$-cr over $k$, then $H$ is a $k$ semisimplification of itself, yielded by the pair $(G, G)$. If $H$ is a $G$-ir subgroup of $G$, then $H$ is the only $k$-semisimplification of $H$ : this shows that not every element of $\mathcal{D}_{k}(H)$ need be a $k$-semisimplification of $H$. In a similar vein, if $P$ and $Q$ are arbitrary $R$-parabolic $k$-subgroups of $G$ and $Q \supseteq P$, then it is easily seen that $Q$ yields a $k$-semisimplification of $P$ if and only if $P^{0}=Q^{0}$. 
Example 4.8. Let $H$ be a subgroup of $G$ and let $P$ be minimal among the R-parabolic $k$-subgroups that contain $H$. Let $L$ be an R-Levi $k$-subgroup of $P$. We claim that $c_{L}(H)$ is L-ir over $k$ (see also [19, Proposition 3.3(a)] and [2, Section 3]); it then follows from Proposition 3.6(ii) that $c_{L}(H)$ is a $k$ semisimplification of $H$. Suppose $c_{L}(H)$ is not $L$-ir: say, $c_{L}(H) \subseteq Q$, where $Q$ is a proper $R$-parabolic $k$-subgroup of $L$. There exist a maximal $k$-torus $T$ of $Q$ and cocharacters $\lambda, \mu \in Y_{k_{s}}(T)$ such that $P=P_{\lambda}$, $L=L_{\lambda}$, and $Q=P_{\mu}$. Now $H \subseteq Q R_{u}(P) \subsetneq P$, and clearly $Q R_{u}(P)$ is $k$-defined. But it is easily checked that $Q R_{u}(P)=P_{m \lambda+\mu}$ for suitably large $m \in \mathbb{N}$ (cf. [2, Lemma 6.2(i)]), so $Q R_{u}(P)$ is an R-parabolic $k$-subgroup of $G$, contradicting the minimality of $P$. Conversely, if $P$ is an $R$-parabolic $k$-subgroup with $R$-Levi $k$-subgroup $L$ such that $P \supseteq H$ and $c_{L}(H)$ is $L$-ir over $k$, then a similar argument shows that $P$ is minimal among the $R$-parabolic $k$-subgroups containing $H$. This proves the claim.

In particular, let $G, H, \lambda$, and $H^{\prime}$ be as in the $\mathrm{GL}_{n}$ example in Section 1. Let $P=P_{\lambda}$ be the parabolic subgroup of block upper triangular matrices with blocks of size $n_{1}, \ldots, n_{r}$ down the leading diagonal. Let $L=L_{\lambda}$ be the subgroup of block diagonal matrices with blocks of size $n_{1}, \ldots, n_{r}$ down the leading diagonal. Since each $n_{i} \times n_{i}$ block yields an irreducible representation of $H^{\prime}:=c_{\lambda}(H), H^{\prime}$ is $L$-ir over $k$, so $P$ is minimal among the R-parabolic $k$-subgroups of $G$ containing $H$; hence $H^{\prime}$ is the $k$-semisimplification of $H$ yielded by $(P, L)$.

Example 4.9. Suppose $\operatorname{char}(k)=0$. Let $H$ be a $k$-subgroup of $G$, and let $P$ be an R-parabolic subgroup of $G$ with $R$-Levi subgroup $L$ such that $P \supseteq H$. Then Corollary 3.5 implies that $c_{L}(H)$ is a $k$-semisimplification of $H$ if and only if $R_{u}(H) \subseteq R_{u}(P)$.

Remark 4.10. Given a reductive k-group $G$ and a subgroup $H$ of $G$, we may (as in Remark 3.2) regard $G$ as a $\bar{k}$-group by forgetting the $k$-structure, so it makes sense to consider the semisimplification (that $i$, the $\bar{k}$-semisimplification) of $H$. The reader is warned that it can happen that $H$ is $G$-cr over $k$ but not $G$-cr, or vice versa (see [2, Example 5.11] and [5, Example 7.22]), so there is no direct relation between the notions of $k$-semisimplification and semisimplification.

\section{Optimality and normal subgroups}

In Example 4.7, we observed that not every element of $\mathcal{D}_{k}(H)$ need be a $k$-semisimplification of $H$. On the other hand, it can happen that $H$ is contained in many different R-parabolic subgroups of $G$, and there may exist many conjugate, but different, $k$-semisimplifications. We now recall two constructions that give under some extra hypotheses a more canonical choice of R-parabolic subgroup yielding a $k$ semisimplification. They apply in particular when $G=\mathrm{GL}_{n}$ (see Example 5.6); this does not seem to be well known even when $k=\bar{k}$.

First construction: Suppose $G$ is connected, $H$ is a subgroup of $G$, and $H$ is not $G$-cr over $k$. We use the theory of spherical buildings (see [18, 19]) and the argument of [3, Proof of Theorem 1.1]. Recall that the spherical building $\Delta_{k}(G)$ of $G$ is a simplicial complex whose simplices are the parabolic $k$-subgroups of $G$, ordered by reverse inclusion (the proper $k$-parabolic subgroups correspond to the non-empty simplices). The apartments of $\Delta_{k}(G)$ are the sets of all $k$-parabolic subgroups of $G$ that contain a fixed maximal split $k$-torus $S$ of $G$. The set $\Sigma$ of parabolic $k$-subgroups $P$ of $G$ such that $P \supseteq H$ is a convex subcomplex of $\Delta_{k}(G)$, and $\Sigma$ is not completely reducible in the sense of [19, Section 2.2] because $H$ is not $G$-cr over $k$ (see [19, Section 3.2.1]). By the Tits Centre Conjecture-see, for example, [4, Section 2.6] and [19, Section 2.4] and the references therein $-\Sigma$ has a so-called 'centre': a proper parabolic $k$-subgroup $P_{c} \in \Sigma$ such that $P_{c}$ is fixed by any building automorphism of $\Delta_{k}(G)$ that stabilizes $\Sigma$. In particular, $P_{c}$ is stabilized by any $k$-automorphism of $G$ that stabilizes $H$.

Lemma 5.1. Let $G, H$, and $\Sigma$ be as above. Let $P_{c}$ be a centre for $\Sigma$ such that $P_{c}$ is not properly contained in any other centre for $\Sigma$. Then $P_{c}$ yields a $k$-semisimplification of $H$.

Proof. Let $\Lambda$ be the set of $k$-parabolic subgroups $Q$ of $G$ such that $Q \subseteq P_{c}$. Fix a Levi $k$-subgroup $L$ of $P_{c}$. We have an inclusion-preserving bijection $\psi$ from $\Lambda$ to $\Delta_{k}(L)$ given by $Q \mapsto Q \cap L$, with inverse given by $R \mapsto R R_{u}\left(P_{c}\right)$. Let $\Sigma_{L}$ be the subset of $\Delta_{k}(L)$ consisting of all the $k$-parabolic subgroups of 
$L$ that contain $c_{L}(H)$. It is clear that $\psi(\Sigma \cap \Lambda)=\Sigma_{L}$. If $\phi$ is a building automorphism of $\Delta_{k}(G)$ that fixes $P_{c}$, then $\phi$ stabilizes $\Lambda$, and we get an automorphism $\phi_{L}$ of $\Delta_{k}(L)$ (as a simplicial complex) given by $\phi_{L}(Q \cap L)=\phi(Q) \cap L$; moreover, if $\phi$ stabilizes $\Sigma$, then $\phi_{L}$ stabilizes $\Sigma_{L}$.

We claim that $\phi_{L}$ is a building automorphism of $\Delta_{k}(L)$. It is enough to show that $\phi_{L}$ maps apartments to apartments. Let $S$ be a maximal split $k$-torus of $L$ (and hence of $G$ ). Since $\phi$ is a building automorphism, there is a maximal split $k$-torus $S^{\prime}$ of $G$ such that for every $k$-parabolic subgroup $Q$ of $G$ that contains $S$, $\phi(Q)$ contains $S^{\prime}$. In particular, $S^{\prime} \subseteq P_{c}$ since $\phi\left(P_{c}\right)=P_{c}$. By Lemma 3.7, there is a $k$-Levi subgroup $L^{\prime}$ of $P_{c}$ such that $S^{\prime} \subseteq L^{\prime}$. By Lemma 2.2(ii), there exists $u \in R_{u}\left(P_{c}\right)(k)$ such that $u S^{\prime} u^{-1} \subseteq L$. Let $R \in \Delta_{k}(L)$ such that $S \subseteq R$ : say, $R=Q \cap L$ for $Q \in \Lambda$. Then $S^{\prime} \subseteq \phi(Q)$. Since $\phi(Q) \subseteq P_{c}, R_{u}(\phi(Q))$ contains $R_{u}\left(P_{c}\right)$, so $u S^{\prime} u^{-1} \subseteq \phi(Q)$. Hence $u S^{\prime} u^{-1} \subseteq \phi(Q) \cap L=\phi_{L}(R)$. This proves the claim.

Now suppose $P_{c}$ does not yield a $k$-semisimplification of $H$. Then $c_{L}(H)$ is not $L$-cr over $k$. By the discussion before the lemma, $\Sigma_{L}$ has a centre $R \subsetneq L$. We have $R=Q \cap L$ for some $Q \in \Lambda$ with $Q \subsetneq P_{c}$. But the results in the previous paragraph imply that $Q$ is a centre for $\Sigma$, contradicting the minimality of $P_{c}$.

Second construction: We allow $G$ to be non-connected again. Suppose the following property holds for a subgroup $H$ of $G$ :

(*) there exists an R-parabolic $k$-subgroup $P$ of $G$ such that $H \subseteq P$ but $H$ is not contained in any R-Levi subgroup - that is, any R-Levi $\bar{k}$-subgroup—of $P$.

This hypothesis implies in particular that $H$ is not $G$-cr over $k$. The construction in [6, Section 5.2] then yields a canonical so-called 'optimal destabilising' R-parabolic $k$-subgroup $P_{\text {opt }}$ of $G$ such that $H \subseteq P_{\text {opt }}$ but $H$ is not contained in any R-Levi subgroup of $P_{\text {opt }}$. If $k$ is perfect then $P_{\text {opt }}$ yields both a $\bar{k}$-semisimplification of $H$ and a $k$-semisimplification of $H$ by [11, Theorem 4.2], but both can fail for general $k$. Moreover, $P_{\text {opt }}$ is stabilized by any $k$-automorphism of $G$ that stabilizes $H$; in particular, if $M$ is a $k$-subgroup of $G$ that normalizes $H$ then $M(k)$ normalizes $P_{\text {opt }}$. See [6, Theorem 5.16] for details.

This construction rests on the notion of an "optimal destabilising cocharacter" due to work of Hesselink [10], Kempf [11] and Rousseau [17]. Roughly speaking, the idea is as follows. Take a generic tuple $\mathbf{h} \in H^{m}$ for $H$. Choose $\mathbf{g} \in G^{m}$ such that $G(k) \cdot \mathbf{g}$ is accessible from $\mathbf{h}$ over $k$ and $G(k) \cdot \mathbf{g}$ is cocharacter-closed over $k$. Set $\mathcal{O}(\mathbf{h})=G(\bar{k}) \cdot \mathbf{g}$; note that $\mathcal{O}(\mathbf{h})$ is uniquely defined by Theorem 2.5. Roughly speaking, we define $\lambda_{\text {opt }} \in Y_{k}(G)$ to be the cocharacter that takes $\mathbf{h}$ into $\mathcal{O}(\mathbf{h})$ as quickly as possible (in an appropriate sense), and we define $P_{\text {opt }}$ to be $P_{\lambda_{\mathrm{opt}}}$. (In fact, we need a slight variationdue to Hesselink - on this construction: rather than taking a single generic tuple $\mathbf{h}$, one considers the action of a cocharacter $\lambda$ on all elements of $H$ at once.) Note that $P_{\text {opt }}$ is not uniquely determined (see [6, Remark 5.22]).

Now suppose that $H$ is a subgroup of $G$ such that $C_{G}(H)$ is $k$-defined. One can show that if $H$ is $G$-cr then $H$ is $G$-cr over $k$ (as previously noted, the converse is false). In fact, we prove a slightly stronger result: if $H$ is not $G$-cr over $k$ then hypothesis (*) holds. To see this, choose a generic tuple $\mathbf{h} \in H^{m}$. We can find $\lambda \in Y_{k}(G)$ such that $\left(P_{\lambda}, L_{\lambda}\right)$ yields a $k$-semisimplification $H^{\prime}$ of $H$; so $G(k) \cdot c_{\lambda}(\mathbf{h})$ is cocharacter-closed over $k$ but $G(k) \cdot \mathbf{h}$ is not. If $H$ is contained in an R-Levi $\bar{k}$-subgroup $L$ of $P_{\lambda}$ then $c_{\lambda}(\mathbf{h})=u \cdot \mathbf{h}$ for some $u \in R_{u}\left(P_{\lambda}\right)$. But then [1, Theorem 7.1] implies that $c_{\lambda}(\mathbf{h})=u_{1} \cdot \mathbf{h}$ for some $u_{1} \in R_{u}\left(P_{\lambda}\right)(k)$, so $G(k) \cdot c_{\lambda}(\mathbf{h})=G(k) \cdot \mathbf{h}$, a contradiction.

Remark 5.2. Let $M$ be a $k$-subgroup of $G$ such that $M$ normalizes $H$, and let $P$ be the R-parabolic subgroup of $G$ obtained from one of the constructions above. Then it is automatic that $M(k)$ normalizes $P$. However, under the extra hypothesis that $H$ is $k$-defined, we can in fact show that $M \subseteq N_{G}(P)$. To see this, one can first extend the field from $k$ to $k_{s}$ and then show that the R-parabolic subgroup obtained from either of the constructions is k-defined (cf. [3, Proof of Theorem 1.1] and [11, Section 4]), and hence coincides with $P$-this implies that $M\left(k_{s}\right)$, and hence $M$, normalizes $P$.

Remark 5.3. There are some limitations on the constructions given above. First, without the hypothesis that $k$ is perfect, it can happen that the subgroup obtained from $P_{\mathrm{opt}}$ is not $G$-cr over $k$, and is 
therefore not a $k$-semisimplification of $H$. (It is, however, $G(\bar{k})$-conjugate to a $k$-semisimplification of $H$.) Second, as yet there is no theory of optimal destabilising subgroups that holds for arbitrary fieldsthis means that we do not know how to define a version of $P_{\text {opt }}$ for a subgroup $H$ that is not $G$-cr over $k$ if $(*)$ does not hold. See [6, Section 1 and Example 5.21] for further discussion of this latter point.

By combining the two constructions above we obtain the following "Clifford theory" result, exploring the link between the semisimplification of a group and a normal subgroup. In the case $k$ is algebraically closed, part (a) is [2, Theorem 3.10].

Theorem 5.4. Let $M$ be a $k$-subgroup of $G$, and let $H$ be a normal $k$-subgroup of $M$. Suppose at least one of the following holds:

(i) $k$ is perfect.

(ii) $G$ is connected.

Then:

(a) If $M$ is $G$-completely reducible over $k$, then $H$ is $G$-completely reducible over $k$.

(b) There is an R-parabolic subgroup $P$ of $G$ such that $M \subseteq P$ and $P$ yields both a $k$-semisimplification of $M$ and $a$-semisimplification of $H$. In particular, there exist $k$-semisimplifications $M^{\prime}$ (respectively, $\left.H^{\prime}\right)$ of $M$ (respectively, of $H$ ) such that $H^{\prime}$ is normal in $M^{\prime}$.

Proof. Suppose $H$ is not $G$-cr over $k$. Choose $P=P_{\text {opt }}$ in case (i) and $P=P_{c}$ in case (ii). Then $M \subseteq N_{G}(P)$ by Remark 5.2. Since $H$ is not contained in any R-Levi $k$-subgroup of $P, H$ is not contained in any R-Levi $k$-subgroup of $N_{G}(P)$ (Lemma 3.8). Hence $M$ is not contained in any R-Levi $k$-subgroup of $N_{G}(P)$. It follows that $M$ is not $G$-cr over $k$. This proves part (a).

For (b), pick $\lambda \in Y_{k}(G)$ such that $\left(P_{\lambda}, L_{\lambda}\right)$ yields a semisimplification $M^{\prime}:=c_{\lambda}(M)$ of $M$. Then $c_{\lambda}(M)$ is $G$-cr over $k$, and $c_{\lambda}(H)$ is normal in $c_{\lambda}(M)$. Now $c_{\lambda}(M)$ and $c_{\lambda}(H)$ satisfy the hypotheses of the theorem, so $c_{\lambda}(H)$ is $G$-cr over $k$ by (a). Hence $\left(P_{\lambda}, L_{\lambda}\right)$ yields a semisimplification $H^{\prime}:=c_{\lambda}(H)$ of $H$ as well, and $H^{\prime}$ is normal in $M^{\prime}$.

Remark 5.5. The hypothesis in part (ii) can be weakened: one only needs to assume that $H \subseteq G^{0}$. In order to make the proof go through, one needs to verify that the first construction above extends to this situation.

Example 5.6. Let $H$ be a $k$-subgroup of $G=\mathrm{GL}_{n}$ such that $H$ is not completely reducible over $k$. Since $H$ is separable, $C_{G}(H)$ is $k$-defined, so $H$ is not $G$-completely reducible; we obtain a parabolic $k$ subgroup $P_{\mathrm{opt}}$ as above which yields a subgroup $H^{\prime}$. We claim that $H^{\prime}$ is a $k$-semisimplification of $H$. For suppose $H^{\prime}$ is not $G$-cr over $k$. Choose $\mathbf{h}, \mathbf{g}$ as above, and let $\mathbf{h}^{\prime}=c_{\lambda_{\mathrm{opt}}}(\mathbf{h})$ (so that $\mathbf{h}^{\prime}$ is a generic tuple for $\left.H^{\prime}\right)$. Since $C_{G}\left(H^{\prime}\right)$ is $k$-defined, hypothesis $(*)$ holds, so we obtain an optimal cocharacter which takes $\mathbf{h}^{\prime}$ out of $G \cdot \mathbf{h}^{\prime}=\mathcal{O}(\mathbf{h})$ and into $\mathcal{O}\left(\mathbf{h}^{\prime}\right)$. But $\mathbf{g}$ is accessible from $\mathbf{h}^{\prime}$ over $k$ by [1, Theorem 4.3(ii)], so $\mathcal{O}\left(\mathbf{h}^{\prime}\right)=\mathcal{O}(\mathbf{h})$, a contradiction.

The parabolic subgroup $P_{\mathrm{opt}}$ is the stabilizer of some flag $\mathcal{F}$ of subspaces of $k^{n}$, and $\mathcal{F}$ does not admit a complementary $H$-stable flag of subspaces of $k^{n}$. By Remark 5.2, $C_{G}(H)$ is a subgroup of $P_{\mathrm{opt}}$-that is, $C_{G}(H)$ stabilizes $\mathcal{F}$-and likewise the normalizer $N_{G}(H)$ stabilizes $\mathcal{F}$ if $N_{G}(H)$ is $k$-defined. If $k$ is perfect then $N_{G}(H)$ is automatically $k$-defined but it need not be $k$-defined in general; see [9] for further discussion.

Remark 5.7. Hesselink gives an example [10, Example 8.5] of a subgroup $H$ of an almost simple group $G$ of type $C_{2}$ such that $P_{\mathrm{opt}}$ is not a minimal centre for $\Sigma$, the subcomplex of the building $\Delta_{k}(G)$ of $G$ consisting of all parabolic subgroups of $G$ that contain $H$. This shows that the two constructions above can yield different R-parabolic subgroups. Nevertheless, the corresponding $k$-semisimplifications of $H$ are $G(k)$-conjugate, thanks to Theorem 4.5. 
Acknowledgments. We are grateful to Brian Lawrence for his questions, which motivated this paper, and for his comments on an earlier draft. We thank the referee for their comments.

We acknowledge support by the DFG Open Access Publication Fund of Ruhr-Universität Bochum.

Conflict of Interest: The authors declare there are no conflicts of interest.

\section{References}

[1] M. Bate, S. Herpel, B. Martin, and G. Röhrle, 'Cocharacter-closure and the rational Hilbert-Mumford theorem', Math. Z. 287(1-2) (2017), 39-72.

[2] M. Bate, B. Martin, and G. Röhrle, 'A geometric approach to complete reducibility', Invent. Math. 161(1) (2005), $177-218$.

[3] M. Bate, B. Martin, and G. Röhrle, 'Complete reducibility and separable field extensions', C. R. Acad. Sci. Paris Ser. I Math. 348 (2010), 495-497.

[4] M. Bate, B. Martin, and G. Röhrle, 'The strong centre conjecture: an invariant theory approach', J. Algebra 372 (2012), 505-530.

[5] M. Bate, B. Martin, G. Röhrle, and R. Tange, 'Complete reducibility and separability', Trans. Amer. Math. Soc. 362(8) (2010), 4283-4311.

[6] M. Bate, B. Martin, G. Röhrle, and R. Tange, 'Closed orbits and uniform S-instability in geometric invariant theory', Trans. Amer. Math. Soc. 365(7) (2013), 3643-3673.

[7] A. Borel, 'Linear algebraic groups', Graduate Texts in Mathematics 126 (Springer-Verlag, 1991).

[8] T. Brüstle, L. Hille, G. Röhrle, and G. Zwara, 'The Bruhat-Chevalley order of parabolic group actions in general linear groups and degeneration for $\Delta$-filtered modules', Adv. Math. 148(2) (1999), 203-242.

[9] S. Herpel and D.I. Stewart, 'On the smoothness of normalisers, the subalgebra structure of modular Lie algebras, and the cohomology of small representations', Doc. Math. 21 (2016), 1-37.

[10] W.H. Hesselink, 'Uniform instability in reductive groups', J. Reine Angew. Math. 303/304 (1978), 74-96.

[11] G.R. Kempf, 'Instability in invariant theory', Ann. Math. 108 (1978), 299-316.

[12] H. Kraft, 'Geometric methods in representation theory', in Representations of Algebras (Puebla, 1980), 180-258, Lecture Notes in Math. 944, (Springer, Berlin-New York, 1982).

[13] B. Lawrence and W. Sawin, 'The Shafarevich conjecture for hypersurfaces in abelian varieties', preprint (2020), https://arxiv.org/abs/2004.09046.

[14] B. Lawrence and A. Venkatesh, 'Diophantine problems and p-adic period mappings', Invent. Math. 221(2020), no. 3, 893-999.

[15] B. Martin, 'Generic stabilisers for actions of reductive groups', Pacific J. Math. 279 (2015), 397-422.

[16] C. Riedtmann, 'Degenerations for representations of quivers with relations', Ann. Sci. École Norm. Sup. (4) 19 (1986), 275-301.

[17] G. Rousseau, 'Immeubles sphériques et théorie des invariants', C. R. Acad. Sci. Paris Sér. A-B 286(5) (1978), A247-A250.

[18] J-P. Serre, 'La notion de complète réductibilité dans les immeubles sphériques et les groupes réductifs', Séminaire au Collège de France, résumé dans [21, pp. 93-98.] (1997).

[19] J-P. Serre, 'Complète réductibilité', Séminaire Bourbaki, 56ème année, 2003-2004, no 932.

[20] T.A. Springer, Linear Algebraic Groups, 2nd edition, Progress in Mathematics 9 (Birkhäuser Boston, Inc., Boston, MA, 1998).

[21] J. Tits, 'Théorie des groupes', Résumé des Cours et Travaux, Annuaire du Collège de France, 97e année, (1996-1997), 89-102.

[22] T. Uchiyama, 'Complete reducibility of subgroups of reductive algebraic groups over nonperfect fields I', J. Algebra $\mathbf{4 6 3}$ (2016), 168-187.

[23] G. Zwara, 'Degenerations for modules over representation-finite algebras', Proc. Amer. Math. Soc. 127 (1999), $1313-1322$.

[24] G. Zwara, 'Degenerations of finite-dimensional modules are given by extensions'. Compositio Math. 121(2) (2000), $205-218$. 